\title{
Path Planning for UAV with Constrained conditions Based on Ant Colony Algorithm
}

\author{
Huiming Zhang a,", Yi Lu, Haizhen Zhu, Zhonghui Xiao, Chunqing Gao \\ Aeronautics and Astronautics Engineering Institute, Air Force Engineering University, Xian 710038, \\ China.
}

a18392639414@163.com

Keywords: UAV; constrained conditions; ant colony algorithm; path planning.

\begin{abstract}
In order to improve UAV's operational efficiency and survival probability, the optimal path of an UAV should be designed before the UAV performs a mission. This paper applies UAV's constrained conditions to the search strategies of ant colony and use a new evaluation method of path's cost. The algorithm's state transformation rules and pheromone updating rules are improved. These make its convergence speed and global searching ability enhanced remarkably. The simulation results show that this method can get a flight path which can avoid threats effectively in a short time and is a more efficient path planning method
\end{abstract}

\section{Introduction}

The flight path planning for UAV is based on the factors such as arrival time, fuel consumption, air defense threat and flight area, so as to plan the optimal or practical flight path for UAV to complete some kind of flight mission ${ }^{[1]}$. The ability of path planning for UAV is necessary to better its fighting performance and implement long-range precision strike. Moreover, path planning is very important to improve the survival ability and the penetration probability of UAV. In recent years, there are a lot of researches on many domestic and foreign scholars in the field of UAV path planning, such as Voronoi algorithm, ant colony algorithm, genetic algorithm, particle swarm algorithm and simulated annealing algorithm. However, these methods are accompanied by many drawbacks and restrictions. For some of the methods, in which the constrained conditions of UAV are ignored, the path generated is always unpractical. Although the ant colony algorithm has good robustness, cooperation and optimization and is easy to combine with other algorithms, it also has some defects, such as long search time and easy to fall into local optimal solution ${ }^{[2]}$.

In order to solve these problems, based on the research of the existing algorithms, we apply the constrained conditions of UAV into the ant colony search strategy in this paper. Meanwhile, the calculation method of threatening cost is bettered, and the convergence speed of the algorithm is improved. Finally, according to characteristics of ant colony algorithm, we optimized its ability of finding global optimal solution by adding guiding factor to state transition rules and dynamical self-adaptive pheromone evaporation coefficient.

\section{Description of Flight path planning}

When penetration and reconnaissance mission is performed by UAV, it is inevitable for them to face the threat from the enemy. The threatening sources include the adverse factors caused by the complex terrain during the flight and the fixed non-attacking threat such as the no fly zone, as well as the radar, air defense positions and other fire threats. In order to simplify the study, it is assumed that the UAV maintains the altitude and speed in the course of flight, and the range of the enemy defense is in a flat area. Consequently, the UAV does not need to plan the unfavorable factors of complex terrain, and the path planning problem can be simplified as a two-dimensional path planning problem. 


\subsection{Constrained Conditions of UAV}

In the course of path planning, it is necessary to take into account the physical limitations of UAV and the specific requirements of the flight mission. The two-dimensional path planning in the horizontal plane mainly includes the following constraints ${ }^{[3]}$.

(1) The minimum distance of every flight segment should be longer than the shortest straight-line flight distance, recorded as $l_{\min }$, for that there must be a straight-line flight distance before the UAV's flight attitude change. The constraint is expressed as $l_{i} \geq l_{\text {min }}$

(2)The sum of each segment flight distance should meet $\sum_{i=0} l_{i} \leq L_{\max }$, where $L_{\max }$ refers to the biggest flight range. This constraint is because that UAV's flight range is limited by the fuel and flight time.

(3) We assume that the maximum turning angle of UAV is $\theta_{\max }$, and $\left(x_{i}, y_{i}\right)$ is position of navigation point and $a_{i}=\left(x_{i+1}-x_{i}, y_{i+1}-y_{i}\right)$ is vector of each flight segment. The constraint of maximum turning angle is expressed as $\left(a_{i}^{T} \cdot a_{i+1}\right) /\left(\left|a_{i}^{T}\right| \cdot\left|a_{i+1}\right|\right) \geq \cos \theta_{\max }$.

(4) There are also constraints for the complex combat field environment which includes no-fly zone and escape zone, threatened area and geographical environment, such as mountains and tall buildings. In the course of path planning, the UAV should fly far away from these areas.

\subsection{Planning Space Modeling and Search Strategy}

In order to facilitate the application of the improved ant colony algorithm to the path planning problem, the path planning space should be rasterized. As shown in Fig.1, the starting point of the UAV flight mission is $S$, the mission target point is $T$, we need to find a short and safe path from $S$ to $T$ point. The corresponding planning region coordinate system is established for the path optimization problem. Point $S$ is selected to be the origin of coordinate. The ray from point $S$ to point $T$ is the horizontal axis and the axis along the clockwise rotation of 90 degrees to form the horizontal ray is vertical axis of the coordinate system. Paralleling to the $X$ axis and $Y$ axis respectively, the two straight line can form a rectangular battlefield area with length of $H$ and width of $W$. By bisecting the battlefield area from direction $X$ and direction $Y$, we can get an area with $m \times n$ grids, where m equals to $W / l_{\min }$, and $\mathrm{n}$ equals to $H / l_{\min }$. When the UAV is in its mission, it flies on grid nodes, whose start point is $(0,0)$ and the terminal is $(m, 0)$. The threatening source, including radar, air defense artillery positions and so on, is seen as circle with a radius of $r$. The five nodes that is closest to the current node are the potential nodes of the next step ${ }^{[4]}$.

In order to get a practical flight path, the constraint condition of UAV is applied to the ant search strategy to delete the unreachable nodes. As is shown in Fig.2, $(a, b)$ is the current node , $(r, s)$ is the previous node and the position of threatening source, whose threatening range is supposed to be $R_{\max }$, is $(p, q)$. In the process of searching for a practical flight path

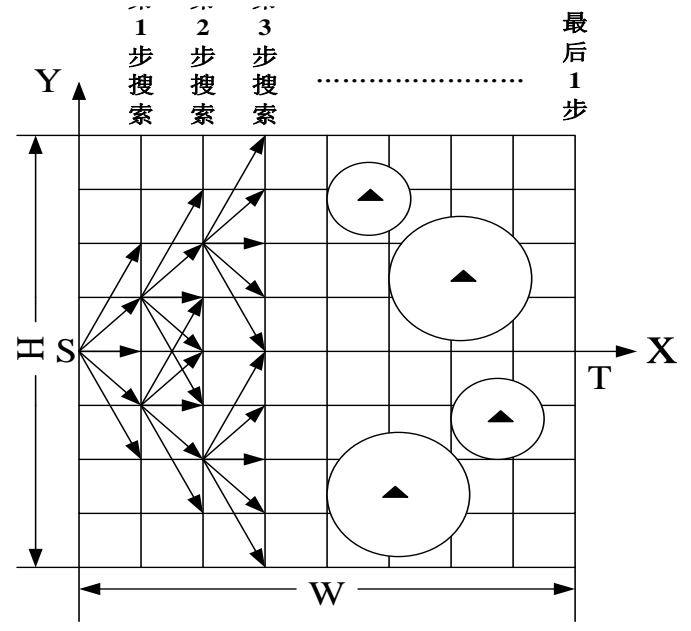

Figure 1. Flight zone model

$(i, j)$, position of candidate node for the path, should meet the following conditions: 
(1)The flight range stretched from $(i, j)$ should not exceed the maximum flight range of the aircraft, which can be expressed as $\sqrt{a^{2}+b^{2}}+\sqrt{(m-a)^{2}+b^{2}} \leq L_{\max }$.

(2)The candidate node should not in the threatened area of the threatening source, i.e. $\sqrt{(p-i)^{2}+(q-j)^{2}} \geq R_{\max }$.

(3)The turning angle to the node cannot be greater than $\theta_{\max }$. The corresponding function is $\left(a_{i}^{T} \cdot a_{i+1}\right) /\left(\left|a_{i}^{T}\right| \cdot\left|a_{i+1}\right|\right) \geq \cos \theta_{\max }$ where $a_{i}=(a-r, b-s)$ and $a_{i+1}=(i-a, j-b)$.

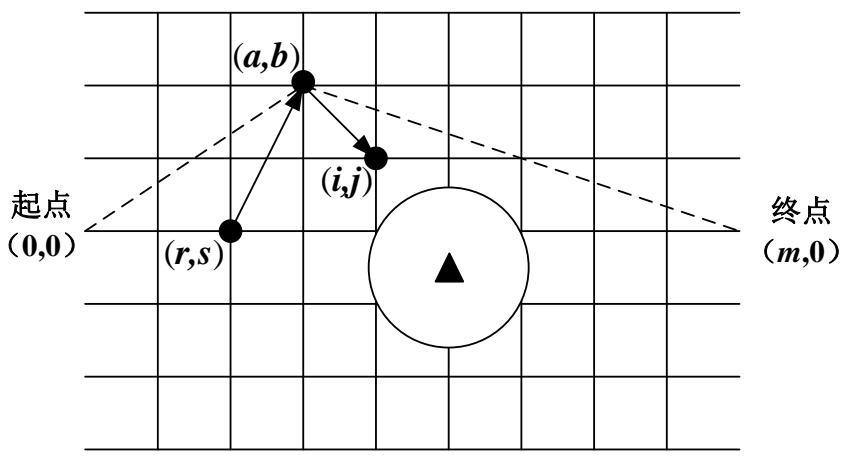

Figure 2. Sketch map of search strategy

\subsection{Flight Path Cost Function}

As we have mentioned above, it is assumed that the UAV maintains the altitude and speed in the course of flight path planning. The cost function is calculated according to the shortest path and the minimum detectable range weighting method and it is used to describe the performance index of the path. Taking into account the detectability index and adopting the acceptable flight range as the mission flight path, the cost function can be expressed as function (1):

$$
\min W=\int_{0}^{L}\left[k w_{t}+\lambda(1-k) w_{f}\right] d l
$$

In the function, $L$ is the flight range and $W$ is generalized cost function. Also, in the function, $w_{t}$ refers to the threatening cost of the flight path, $w_{f}$ represents for fuel cost and $\lambda$ is balance coefficient which is usually expressed as $\left[w_{t} / w_{f}\right]$ and used to adjust dimensional level of threat cost and fuel consumption.

In the process of searching for next nodes, according to the formula (1), we calculate the weight of each edge of the grid graph. Take the $\mathrm{i}$-th edge of the grid as an example, We can get function (2).

$$
w_{i}=k w_{t, i}+(1-k) w_{f, i} \quad 0 \leq k \leq 1
$$

In the function, $w_{i}$ is generalized cost of the i-th edge, $w_{t, i}$ and $w_{f, i}$ is threat cost and fuel cost of the i-th edge respectively.

The threatening model can be simplified by the assumption that the enemy radar and missile positions in the restricted area are the same and have no relation to each other. The threatening index is directly proportional to $1 / d^{4}$, where $d$ refers to the distance between an aircraft and a radar or missile position within the restricted area. The existing researches in the calculation of a path section of the threat cost adopt the method that selecting a number of equidistant points on the path to find the average value and multiply the length of the path segment. Nevertheless, this method is too cumbersome which increases the planning time, and does not conform to the actual battlefield. In this paper, when the UAV is flying in the i-th flight path segment, we just calculate the threat cost of the nearest threat point on the track. As is shown in Fig.3, the threat cost between A and B can be calculated by function (3).

$$
w_{t, j}=\sum_{j=1}^{N} \frac{1}{d_{i, j}^{4}}
$$

$N$ is the number of threatening areas of radar and missile positions and $d_{i, j}$ refers to the distance from the closest point to threat $j$ in the i-th flight path segment. 


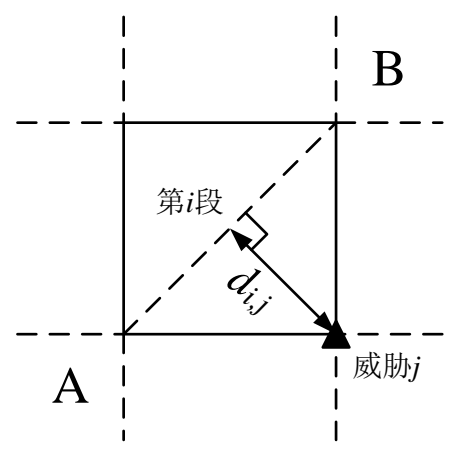

Figure 3. Sketch map of threat cost calculation

If the speed maintains at the same level, fuel consumption is proportional to the flight range, so we can simply think that $w_{f}=L$ and $w_{f, i}=L_{i}$.

\section{Improved Ant Colony Algorithm}

Ant colony algorithm is simulating foraging activities of ant colony that from nest to food source. The ants transmit information to others through pheromone, and it is treated as a signal to influence the followers' actions. Afterwards, the previous pheromone will be strengthened by the followers, and so on. From a mathematical point of view, the path of ant colony from nest to food source composes the smallest tree and the mission is to search the path with the smallest energy consumption.

The ant colony algorithm is briefly as follows: first of all the nodes are given appropriate initial regional grid value, forming the initial pheromone matrix. Then the ants are all placed in the starting point with the same target direction. In the process, each ant selects adjacent feasible node according to the feasible node state transition rule until all the ants reach the target. This is completion of a cycle. After a cycle is completed, global pheromone will be updated on the basis of the ant path. The pheromone of the node that has not been passed is volatilized, and the other nodes are strengthened according to the transition rules of pheromone. The colony will repeat this process until the optimal path is obtained.

\subsection{State transition rule}

In order to increase the predictability of the target node, we introduce the target node pheromone guiding factor to the current node in this paper. For instance node $(i, j)$, the guiding factor can be calculated by (4), where $d_{(i, j)}$ is the distance from node $(i, j)$ to the terminal.

$$
\lambda_{(i, j)}=1 / d_{(i, j)}
$$

It is obvious that the guiding is greater if the candidate node is nearer to the terminal and vice versa. Therefore, in the state transition strategy, the introduction of the guidance factor can effectively reduce the blindness of the ant search, and enable the ant to search in the direction of the terminal.

According to the cost of the edge between the two grid nodes and the pheromone concentration of the neighboring nodes, the ant $k(k=1,2, \cdots, m)$ decides the next node. At the moment $t$, we suppose that the ant is at any node which says $(a, b)$, the transition possibility of the ant from node $(a, b)$ to node $(i, j)$ is expressed as function (2).

$$
p_{i j}^{k}= \begin{cases}\frac{\left[\tau_{(i, j)}(t)\right]^{\alpha} \cdot\left[\eta_{(i, j)}(t)\right]^{\beta} \cdot\left[\lambda_{(i, j)}(t)\right]^{\gamma}}{\sum_{(x, y) \in \text { allow }(a, b)}\left[\tau_{(x, y)}(t)\right]^{\alpha} \cdot\left[\eta_{(x, y)}(t)\right]^{\beta} \cdot\left[\lambda_{(x, y)}(t)\right]^{\gamma}} & (x, y) \notin \operatorname{allow}(a, b) \\ 0 & (x, y) \in \operatorname{allow}(a, b)\end{cases}
$$

In $(5), \tau_{(i, j)}(t)$ is the pheromone concentration of node $(i, j), \eta_{(i, j)}(t)$ refers to the heuristic function and $\eta_{(i, j)}(t)=1 / w_{(i, j)}$, where $w_{(i, j)}$ means transition expectation degree of the edge from node $(a, b)$ to node $(i, j) \cdot \lambda_{(i, j)}(t)$ represents guiding factor and allow $(a, b)$ is the candidate sets of nodes when the ant is at node $(a, b)$. As for $\alpha, \beta, \gamma$, they are importance degree parameter of pheromone, heuristic factor 
and guiding factor. Besides, simulation results show that the value of $\beta$ and $\gamma$ should not be too large, otherwise the algorithm is easy to fall into stagnation.

\subsection{Pheromone Update rule}

The interaction mechanism is adopted for local pheromone updating and global pheromone updating in this paper. The local pheromone updating means that when the ant passes the node, the pheromone of the node is reduced, and the purpose of local updating is to increase the probability of searching the node that didn't passed, so as to achieve the purpose of global search. Local pheromone updating goes with the searching of ant and the updating function is expressed as function (6).

$\tau_{(i, j)}=(1-\xi) \tau_{(i, j)}$

In the function, $\tau_{(i, j)}$ is the value of pheromone on the node and $\xi$ refers to attenuation coefficient of pheromone.

Global updating means that when the ant completes a path search, the cost of the path is taken as the evaluation value, and the shortest path is selected from the path set to increase the pheromone value of each node on the shortest path. The pheromone updating process is showed in function (7).

$$
\left\{\begin{array}{l}
\tau_{(i, j)}=(1-\rho) \tau_{(i, j)}+\rho \Delta \tau_{(i, j)} \\
\Delta \tau_{(i, j)}=\frac{Q}{\min (W(m))}
\end{array}\right.
$$

In (7), $W(m)$ is the cost value of the m-th ant passing the path, $\rho$ is pheromone evaporating factor and $Q$ is a constant which means the total amount of pheromone released by the ant in on cycle.

In the optimization process of ant colony algorithm, after a certain number of iterations, the evolution direction tends to maintain steady. The optimal solution algorithm is suspected in local optimum. At this situation, parameter $\rho$ can be adaptively adjusted, so as to improve the globalization of algorithm and avoid the local optimal solution.

We suppose that the initial value of pheromone evaporation factor $\rho$ is $\rho\left(t_{0}\right)=1$. When the optimal solution obtained by ant colony algorithm has no obvious change in many cycles, the adaptive adjustment rule is expressed in (8).

$$
\rho(t)=\left\{\begin{array}{l}
0.9 \rho(t-1), \text { if } 0.9 \rho(t-1) \geq \rho_{\text {min }} \\
\rho_{\text {min }}, \text { otherwise }
\end{array}\right.
$$

$\rho_{\min }$ is the minimum pheromone evaporation factor. In this paper, value of $\rho_{\min }$ is set to 0.2 to avoid that $\rho$ is too small to reduce the convergence speed of the algorithm.

\section{Case Study}

The combating area is supposed to be $400 \mathrm{Km} \times 400 \mathrm{Km}$, minimum step size of UAV is $2 \mathrm{Km}$. So the planning area can be divided into $200 \times 200$ grids, the starting point is $(0,0)$, the terminal node is at $(200,0)$ and the maximum turning angle of UAV is $\theta_{\max }=60^{\circ}$.It is known by the prior battlefield investigation that the distribution and the range of the threatening source position in the combating area is shown in table 1. Besides, the other parameters are as follows: $\alpha=3, \beta=5, \gamma=4, k=0.2, N_{\max }=100$, $m=30, \xi=0.5, \rho=0.9, \rho_{\min }=0.2, Q=100$, where $N_{\max }$ refers to maximum number of iterations allowed and $m$ is the number of ants.

Table 1. Threat distribution table

\begin{tabular}{ccc}
\hline Serial number & Position coordinate & Threatening range $(\mathrm{Km})$ \\
\hline a & $(30,-30)$ & 30 \\
b & $(50,0)$ & 30 \\
c & $(100,0)$ & 40 \\
d & $(150,10)$ & 30 \\
e & $(160,-30)$ & 40 \\
\hline
\end{tabular}

In this paper, Matlab7.6 software is used to simulate the basic ant colony algorithm and the improved ant colony algorithm respectively. At the same time, five simulation experiments are carried 
out according to the initialization parameters. The minimum comprehensive cost and running time for each experiment are recorded, and the results are shown in Table 2 and Table 3 respectively.

Table 2. Results comparison of comprehensive flight path cost

\begin{tabular}{ccc}
\hline $\begin{array}{c}\text { Serial number of } \\
\text { experiments }\end{array}$ & $\begin{array}{c}\text { Comprehensive flight path cost of basic } \\
\text { ant colony algorithm }\end{array}$ & $\begin{array}{c}\text { Comprehensive flight path cost of } \\
\text { optimized ant colony algorithm }\end{array}$ \\
\hline 1 & 232.9261 & 224.6528 \\
2 & 235.4894 & 229.2179 \\
3 & 234.0332 & 227.1402 \\
4 & 232.8895 & 227.2564 \\
5 & 233.7408 & 221.3544 \\
Maximum value & 232.8895 & 221.3544 \\
Average value & 233.8200 & 225.9200 \\
\hline \multicolumn{4}{c}{ Terial number of } & Ruble 3. Comparison of algorithm run time \\
experiments & \multicolumn{3}{c}{ of basic ant colony algorithm } & Run time of optimized ant colony \\
\hline 1 & $34.8138 \mathrm{~s}$ & algorithm \\
\hline 3 & $34.7394 \mathrm{~s}$ & $25.1563 \mathrm{~s}$ \\
4 & $34.6901 \mathrm{~s}$ & $26.0108 \mathrm{~s}$ \\
5 & $34.4306 \mathrm{~s}$ & $25.2112 \mathrm{~s}$ \\
Maximum value & $34.6669 \mathrm{~s}$ & $25.5283 \mathrm{~s}$ \\
Average value & $34.4306 \mathrm{~s}$ & $25.2853 \mathrm{~s}$ \\
\hline
\end{tabular}

By comparing the simulation results in Table 2 and table 3, it can be seen that in the five experiments, the improved ant colony algorithm is superior to the basic ant colony algorithm in the average track cost and the average run time. Therefore, it is obvious that the improved ant colony algorithm can not only find a better solution, but also shorten the running time of the algorithm. Fig.4 and Fig.5 are the best track simulation results of the two algorithms in the five simulation experiments. Figure 6 and Figure 7 is the sketch map of the optimal path of the two algorithms in the five simulation experiments respectively.

It can be informed from Figure 6 and Figure 7 that ,at several singular nodes, the turning angle of the flight path planned by basic ant colony algorithm exceeds the maximum turning angle of the UAV, so it is an unpractical path. While, by applying the constraints to searching strategies, the flight path calculated by optimized ant colony algorithm, excluding the singular nodes, is practical. Moreover, running time of the optimized ant colony algorithm is shortened and turning angle did not exceed the maximum turning angle of the UAV

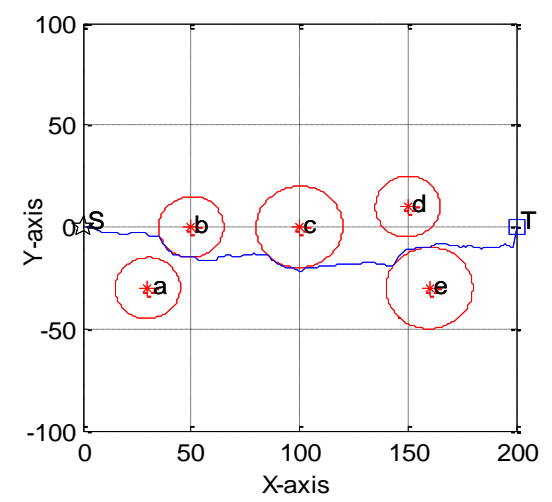

Figure 4. Simulation of basic ant colony algorithm 


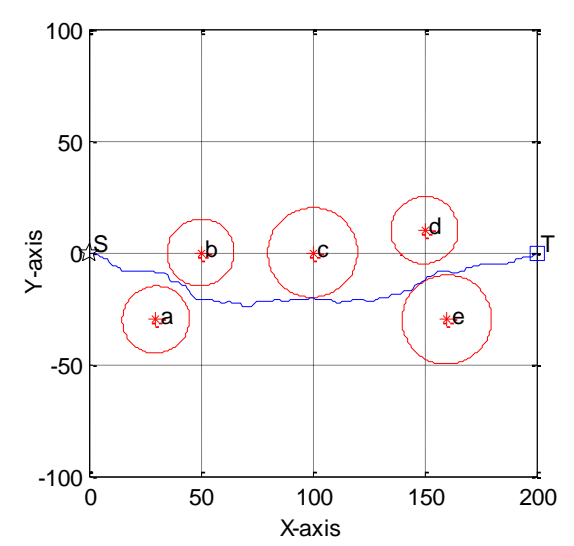

Figure 5. Simulation of optimized ant colony algorithm

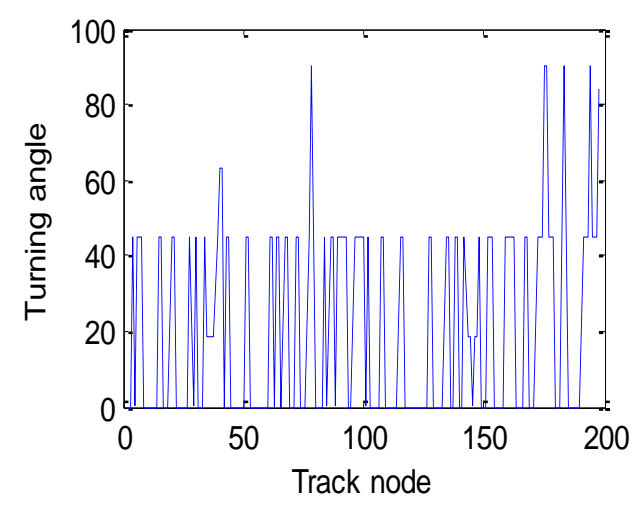

Figure 6. Flight path turning angle using basic ant colony algorithm

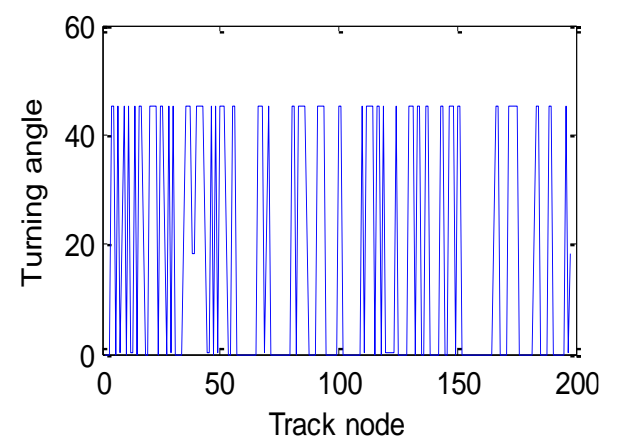

Figure 7. Flight path turning angle using optimized ant colony algorithm

\section{Summary}

In this paper, based on the basic ant colony algorithm, the constraints of UAV flight path planning are applied to the ant colony searching strategy, excluding the unpractical nodes, running time of the algorithm is shorten and the speed of planning is improved. Also, the guiding factor is added in the state transition rule, improving the convergence speed of the algorithm. Besides, the pheromone is updated using local and global update combination method and self-adaptive adjustment of pheromone evaporation coefficient is adopted, which improves the ability of global searching and avoids the premature fall into the local optimal solution. the optimized ant colony algorithm would effectively guarantee the safety of UAV and improve the operational efficiency of the UAV. The simulation results show that the proposed method, in this paper, is of high efficiency, and is able to plan a UAV practical path in a relatively short period of time, which verified the feasibility and validity of the method. 


\section{References}

[1] Changwen Zheng, Ping Yan, Mingyue Ding. Research status and trend of route planning for flying vehicles[J]. Journal of Astronautics. Vol.28(2007)No.6,p.1441-1444.

[2] Qi Wu, Guangzhen Pan, Jiangtao Yang. Route planning of UAV based on voronoi diagram and dynamic and adaptive ant colony algorithm[J]. Computer Measurement and Control. Vol.2 2 (2014) No.9, p.3037-3040.

[3] Shiyong Li, Dan Yang. Route planning of cruise missile based on improved ant colony algorithm[J]. Journal of Astronautics. Vol.28(2007)No.4,p.903-907.

[4] Biwei Tang, Qun Fang, Zhanxia Zhu. Effective 2D route planning of UAV based on improved ant colony algorithm $\mathrm{m}[\mathrm{J}]$. Journal of Northwestern Polytechnical University. Vol.31(2013)No.5,p.683-687.

[5] Huaguang Zhu, Li Liu, Yong Zhu. Route planning for UAV with practicable overload constrained based on ant algorithm. Chinese Journal of Scientific Instrument. Vol.29(2008)No.4,p.260-264.

[6] Shou Liu, Yiyu Chen, Bin Zhong. Optimal path planning of unmanned aerial vehicle based on adaptive ant colony algorithm[J]. Journal of Ordnance Engineering College. Vol.28(2016)No.1,p.46-51.

[7] Zhonghua Hu, Min Zhao, Shihao Liu. Unmanned aerial vehicle flight path planning based on adaptive ant colony optimization algorithm[J]. Computer Integrated Manufacturing Systems. Vol.18 (2012) No.3, p.560-565.

[8] Yu Xiong, Yuedong Yao. Route planning of UAV based on improved ant colony algorithm[J]. Computer and Digital Engineering. Vol.38(2010)No.7,p.41-44. 